\title{
Giant fibroadenoma of breast: a diagnostic dilemma in a middle aged woman
}

\begin{abstract}
Giant breast masses are entities of great concern as they continue to pose a diagnostic as well as a therapeutic challenge. We present a case of giant fibroadenoma of the right breast in a 35-year-old woman which simulated as a phyllodes tumor, creating a diagnostic conundrum.
\end{abstract}

Keywords: breast, cytology, giant fibroadenoma, middle aged woman, phyllodes tumor, histopathology, breast conserving surgery

\author{
Volume 2 Issue 4 - 2017 \\ Sonam Sharma,' Bhanu Pratap Rana ${ }^{2}$ \\ 'Department of Pathology, Pandit Bhagwat Dayal Sharma \\ University of Health Sciences, India \\ ${ }^{2}$ Department of Surgery, Pandit Bhagwat Dayal Sharma \\ University of Health Sciences, India
}

\begin{abstract}
Correspondence: Sonam Sharma, Department of Pathology, Kalpana Chawla Government Medical College, Karnal, Haryana, India,Tel 999984I393, Email drsonamsharma@gmail.com
\end{abstract}

Received: June 21, 2017 | Published:September 01, 2017

\section{Introduction}

Fibroadenoma is the commonest solid benign lesion that can exist as a solitary mass or multiple masses in the breasts. ${ }^{1}$ It is encountered in women usually before the age of 30 (commonly between 10-18 years of age), ${ }^{2}$ although its occurrence in postmenopausal women, especially those receiving estrogen replacement therapy has been documented. ${ }^{3}$ Giant (juvenile or cellular) fibroadenoma is a rare variant of fibroadenoma accounting for 0.5-2per cent of all cases of fibroadenomas ${ }^{4}$ and is characterized by its large size and rapid growth. ${ }^{5}$ This subtype has been also been defined as fibroadenoma with size larger than $5 \mathrm{~cm}$ and/or weight of more than 500 grams. ${ }^{6}$ Most commonly it is seen in females of Afro-Caribbean or East Asian descent and have a bimodal age distribution with occurrence typically either in adolescent or premenopausal women. ${ }^{7}$ Increased estrogen stimulus and receptor sensitivity and a decrease in estrogen antagonist levels during puberty are thought to be the basis of its etiology. 2,8 This rare entity is of great importance as this may not only cause asymmetrical breasts, stretching and displacement of the nipple-areola complex, congestion and ulceration of skin by centrifugal pressure but also it has to be differentiated from other giant breast masses as all these have a different therapeutic approach and prognosis. We herein report one such unusual case of giant fibroadenoma of the right breast in a 35-year-old woman creating a diagnostic dilemma as it mimicked a phyllodes tumor.

\section{Case presentation}

A 35-year-old woman came to the surgical outpatient department of our hospital with the chief complaints of asymmetrical breasts and a large lump in the right breast. She gave a history that the lump was first noticed around 10 years back in the form of small nodule, which increased progressively in size with a rapid enlargement over a period of last 6 months. She also complained of associated dragging non-radiating type of pain in the right breast since last 15 days. There was no history of breast trauma, fever, anorexia or weight loss. Her past, medical and family history was non-contributory. Her menstrual cycles were regular and she did not take any hormonal therapy. On physical examination, the right breast was slightly bigger than the left breast and the right nipple-areola complex was displaced laterally (Figure 1A). On palpation, there was a lump in the right breast which measured $12 \times 6 \mathrm{~cm}$ in size and was located in the right upper and lower inner quadrant . It was firm in consistency, non-tender, freely mobile within the breast tissue and free from the chest wall. The overlying skin was tense and shiny with prominent distended superficial veins (Figure 1B). There was no skin ulceration or any nipple discharge. Her systemic examination, the left breast and bilateral axilla were within normal limits. Based on the patient's age, history and the breast examination, a clinical diagnosis of phyllodes tumor was made. Her routine hematological, biochemical and microbiological parameters were within normal limits.

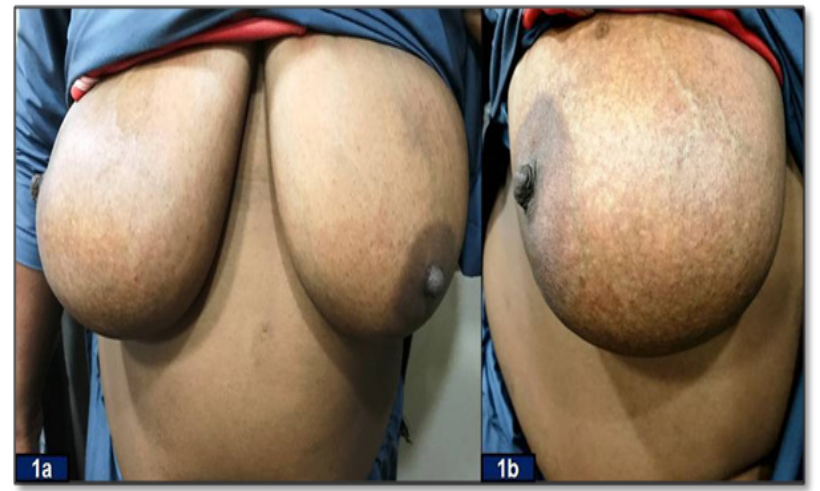

Figure I

(A) Asymmetrical breasts and deviation of nipple-areola complex of the right breast on clinical inspection.

(B) Distended tortuous veins on the overlying skin of the right breast lump.

Chest X- ray was normal. Ultrasonography of the right breast showed a well-circumscribed hypoechoic lesion of $12 \times 7.5 \mathrm{~cm}$ size in inner half of the right breast with smooth margins and broad base. A hyperechoic nodule of $2.1 \times 2 \mathrm{~cm}$ was noted within this lesion and there was minimal increase in its vascularity. There was no evidence of enlarged intra mammary or axillary lymph nodes. The retro mammary region consisting of fat, ribs and muscle was normal. The skin thickness and the nipple as well as subareolar tissue was normal. Based on these radiological findings, a differential diagnosis of phyllodes tumor and giant fibrodenoma were made. Fine-needle aspiration cytology 
(FNAC) of the breast lump was performed. Smears were drawn and stained with May-Grunwald-Giemsa (MGG) stain. The cytology smears showed tightly cohesive monolayered sheets and aggregates of benign ductal epithelial cells with myoepithelial cells scattered in between these epithelial fragments. In the background numerous single bare bipolar nuclei were seen (Figure 2A \& 2B). Few stromal fragments along with spindle shaped cells having elongated nuclei and adipocytes were also evident. On the basis of these cytomorphological features, a diagnosis of a benign proliferative lesion suggestive of fibroadenoma was made.

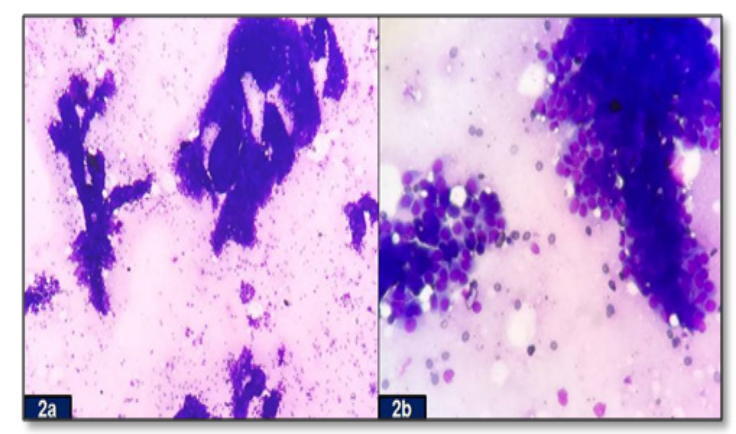

Figure 2

(A) Cell-rich smear of elongated, branching stag-horn like fragments of ductal epithelial cells and numerous single bipolar nuclei in the background (MGG stain, $\times 100$ ).

\section{(B) Aggregates of cohesive epithelial cells (MGG stain, $\times 400$ ).}

The patient underwent total excision of right breast lump while the normal breast tissue, nipple, and areola were preserved. The resected specimen was sent for the histopathological examination. The right-sided excised lump measured $12 \times 7 \times 5 \mathrm{~cm}$ and it weighed $650 \mathrm{gm}$. External surface of the lump was bosselated and it was well circumscribed and encapsulated. On cut section, multiple grey white nodules with slit-like spaces in between were seen. Microscopic hematoxylin and eosin (H\&E) stained sections revealed wellencapsulated tumor with hyperplasia of both epithelial and stromal components, forming both intracanalicular and pericanalicular growth pattern (Figure 3). There was no leaf-like growth pattern and cytological atypia despite of stromal cellularity. The final histopathological diagnosis of giant fibroadenoma of the right breast was rendered. Her postoperative recovery period was uneventful. The patient is on regular follow up and is doing well so far with no loco regional recurrence.

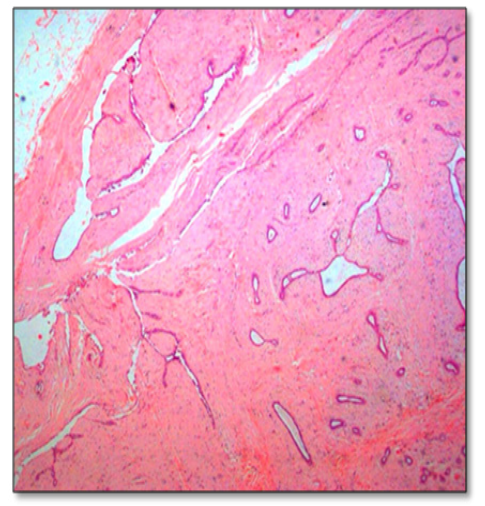

Figure 3 Histopathological sections exhibiting a well-circumscribed, encapsulated tumor with biphasic proliferation of both glandular and stromal cells $(\mathrm{H} \& \mathrm{E}, \mathrm{x} \mid 00)$.

\section{Discussion}

Breast lesions are a common problem in females that are encountered routinely in day today practice. Among them, giant breast masses are quite interesting because of their rarity and unpredictable behaviour. They usually give rise to a suspicion of malignancy owing to their rapid growth, associated skin congestion/ulceration and tendency to recur. ${ }^{9,10}$ A wide variety of breast conditions such as fibroadenoma, phyllodes tumor, virginal hypertrophy, lipoma, hamartoma, cyst, abscess and carcinoma can result in solitary or multiple giant masses. It is important to distinguish these pathological entities preoperatively as the treatment modalities and the prognosis differ quite significantly in all these conditions. Some of the lesions are treated by mastectomy, while some other lesion may require only local excision, aspiration, or conservative management. ${ }^{11,12}$ However, owing to the varied histological features seen in these giant breast masses, there have been varying interpretations and diagnosis. This has led to inappropriate and at times unnecessarily radical surgical therapy. ${ }^{5}$

The current case posed a diagnostic challenge as it masqueraded as a phyllodes tumor. Similar diagnostic dilemma has been documented in the literature in females of different age groups. ${ }^{13-16}$ Phyllodes tumor is a very rare breast tumor and constitutes $0.3-1.0 \%$ of mammary tumors and $2-3 \%$ of fibroepithelial neoplasms of the breast. It usually occurs in the 4th decade and rarely during puberty. It exists in benign, borderline and malignant forms. ${ }^{8,17}$ Both giant fibroadenoma and phyllodes tumor arise from intralobular stroma, ${ }^{18}$ although on clonal analysis it has been found that a giant fibroadenoma is a polyclonal fibroadenoma that has attained an immense size and is different from the monoclonal phyllodes tumor. ${ }^{19}$ Owing to their genesis, there is a close resemblance between the two entities in terms of their clinical, radiological, cytological and histopathological appearances, like in our case. Nevertheless, it is very important to differentiate the two pathologies preoperatively as they have a different therapeutic approach. $^{20}$

Another important differential of giant fibroadenoma is the breast carcinoma, as it is the most common cancer especially among Indian women replacing cervical cancer. ${ }^{21}$ Although, malignant breast tumors are rare in young women however, it has been documented that though the risk of breast cancer increases with age but for women of 40 years of age or younger, the chances of developing breast cancer over the next 10 years is less than $2 \%$ and often a search for a genetic component to the cancer is indicated in such cases. The younger women tend to present in advanced stage with a fast growing, higher grade and estrogen receptor negative tumor. ${ }^{22,23}$ In the current case, none of the features of malignancy were evident on clinical examination, radiology and cytology, hence the possibility of breast cancer was ruled out.

Giant fibroadenoma in a 35-year-old female also needs to be differentiated from other breast conditions like lipoma. abscess, hamartomas and cysts. Giant lipoma can cause soft, mobile mass in unilateral breast. Histopathology reveals encapsulated nodules of mature adipose tissue. Breast abscesses can cause sudden and rapid growth in the breast with pain and redness. Histological examination in such cases usually show focal collection of polymorphs with necrotic material in the lobules. Hamartomas on histopathology reveals an admixture of ducts, lobules, fibrous stroma, and adipose tissue in varying proportions [24]. Large cysts of fibrocystic disease as well as parasitic cysts can also present as giant breast masses [25]. 
The diagnosis of giant fibroadenoma is usually difficult initially as the physical examination of the breasts is not diagnostic because of the converging clinical presentation of large mass leading to assymetrical breasts, skin changes or deviation of nipple areola complex. Ultrasonography and mammography are the two basic techniques for routine imaging in the diagnosis of breast diseases whereas Magnetic Resonance Imaging (MRI) allows exact evaluation of size and location. However, in similarity to our case, authors have reported that both the physical examination and standard radiographic imaging usually fail to pinpoint the exact diagnosis in many cases. ${ }^{13,26}$ Preoperative cytological examination is essential as it plays a pivotal role in clinching the diagnosis in most of the cases, as seen in our case. However, sometimes the features of fibroadenomas and benign or borderline phyllodes tumor overlap each other, thereby making the diagnosis difficult on FNAC ${ }^{15,27}$ However, it is the histopathology which is considered to be the gold standard as it is not only required for the definite diagnosis but it also helps in differential diagnosis of giant breast masses, thereby allowing timely intervention and appropriate treatment in such cases.

Surgical excision is usually needed for giant fibroadenoma if the mass continues to grow or causes cosmetic concerns. Complete local excision with sparing of breast tissue, as done in our case is often the treatment modality of choice. To excise it, numerous surgical techniques, such as conventional local excision, reduction mammoplasty, or inframammary excision, round-block technique have been reported, ${ }^{28,29}$ The biological behavior of the giant fibroadenoma is usually benign. It may recur after complete excision however, the chances of recurrence become less after third decade of life. ${ }^{30}$

\section{Conclusion}

Giant fibroadenoma of the breast is a rare tumor which can occur at any age in a female and can have varied presentations, thereby creating a diagnostic dilemma. Therefore, this entity should always be kept in the mind while dealing with a case of giant breast mass, irrespective of the age. Detailed clinical examination, radiology, preoperative cytology of all large breast lumps is important before deciding on appropriate surgical management. However, it is the histopathology which is important for a definite diagnosis in such cases. Breast conserving/sparing surgery is the treatment of choice for giant fibroadenoma of the breast owing to its benign nature and a very low recurrence rate after excision.

\section{Conflict of interest}

No financial interest or any conflict of interest exists.

\section{Acknowledgements}

None.

\section{References}

1. Greenberg R, Skornick Y, Kaplan O. Management of breast fibroadenomas. J Gen Intern Med. 1998;13(9):640-645.

2. Lo Martire N, Nibid A, Farello G, et al. Giant fibroadenoma of the breast in an adolescent: a case report. Ann Ital Chir. 2002;73(6):631-634.

3. Yilmaz E, Sal S, Lebe B. Differentiation of phyllodes tumors versus fibroadenomas: Mammographic and sonographic feature. Acta Radiologica. 2002;43(1):34-39.

4. Baxi M, Agarwal A, Mishra A, et al. Multiple bilateral giant juvenile fibroadenomas of breast. Eur J Surg. 2000;166(10):828-830.
5. Arowolo OA, Akinkuolie AA, Adisa AO, et al. Giant fibroadenoma presenting like fungating breast cancer in a Nigerian teenager. Afr Health Sci. 2013;13(1):162-165.

6. Wechselberger G, Schoeller T, Piza-Katzer H. Juvenile fibroadenoma of the breast. Surgery. 2002;132(1):106-107.

7. Hanna RM, Ashebu SD. Giant fibroadenoma of the breast in an Arab population. Australas Radiol. 2002;46(3):252-256.

8. Marchant DJ. Benign breast disease. Obstet Gynecol Clin North Am. 2002;29(1):1-20.

9. Raganoonan C, Fairbain JK, Williams S, et al. Giant breast tumours of adolescence. Aust N Z J Surg. 1987;57(4):243-247.

10. Carl D, Patel V. Surgical problems in the management of the breast tumour. Am J Obstet Gynecol. 1985;152:1010-1015.

11. Muttarak M, Chaiwun B. Imaging of giant breast masses with pathological correlation. Singapore Med J. 2004;45(3):132-139.

12. Uygur F, Yigitler C. Rare juvenile giant fibroadenoma. J Breast Health. 2009;5:164-166.

13. Rattan K, Kumar S, Dhull A, et al. Giant fibroadenoma mimicking phylloides tumor in a young female: A cytological dilemma. The Internet Journal of Third World Medicine. 2007;6(2):1-4.

14. Bansal M, Kaur K, Singh J, et al. Giant fibroadenoma mimicking phylloides tumor: A rare occurrence in a postmenopausal female. $J$ Evolution Med Dent Sci. 2015.35(4):6148-6152.

15. Shah B, Shah BB, Sharma K, et al. Giant fibroadenoma of the breast mimicking phyllodes tumor in an adult female: emphasizing the role of cytology in the diagnosis. Int J Stud Res. 2014;4(2):44-45.

16. Alhefdhi A. Giant fibroadenoma mimicking phylleoyeds tumor. Obstet Gynecol Int J. 2017;6(4):00213.

17. Gunasekaran G, Naik D, Sharma S, et al. Recurrent phyllodes tumor of breast transforming to a fibrosarcoma. Int Surg J. 2014;1(3):173-176.

18. Park CA, David LR, Argenta LC. Breast asymmetry: presentation of a giant fibroadenoma. Breast J. 2006;12(5):451-461.

19. Noguchi S, Aihara T, Motomura K, et al. Demonstration of polyclonal origin of giant fibroadenoma of the breast. Virchows Archiv A Pathol Anat. 1995;427(3):343-347.

20. Krishnamurthy S, Ashfaq R, Shin HJ, et al. Distinction of phyllodes tumor from fibroadenoma: A reappraisal of an old problem. Cancer. 2000;90(6):342-349.

21. Sharma S, Chintamani, Khambra P, et al. Synchronous Oral and Breast Malignancies: A Rare Presentation. Indian J Surg Oncol. 2017;8(3):426-429.

22. American Cancer Society. Breast Cancer Facts \& Figures. 2013;20132014.

23. Freedman RA, Partridge AH. Management of breast cancer in very young women. Breast. 2013;2:S176-179.

24. Issam M. Giant fibroadenoma. Case report and review of literature. Bas J Surg. 2006;12:1-4.

25. Sharma S, Singhal S, Sharma R. Cysticercosis of Breast Masquerading as Fibroadenoma. Rec Adv Path Lab Med. 2016;2(2):7-9.

26. Page JE, Williams JE. The radiological features of phylloides tumour of the breast with clinico-pathological correlation. Clin Radiol. 1991;44(1):8-12.

27. Bandyopadhyay R, Nag D, Modal SK, et al. Distinction of phylloides tumor from fibroadenoma: Cytologist's perspective. $J$ Cytol. 2010;27(2):59-62. 
28. Chang DS, McGrath MH. Management of benign tumors of the adolescent breast. Plast Reconstr Surg. 2007;120(1):13e-19e.

29. Lai HW, Lee CW, Li YH, et al. Juvenile giant fibroadenomas with apparent breast asymmetry successfully managed by round-block technique. JPRAS Open. 2015;(6):40-43.
30. Mukhopadhyay M, Patra R, Mondal S, et al. Bilateral giant juvenile fibroadenoma of breasts. J Indian Assoc Pediatr Surg. 2009;14(2):6869. 